\title{
From Principles to Practice
}

\section{Handbook of Psychiatric Education, Second Edition. Edited by Donna M. Sudak, M.D. American Psychiatric Association Publishing; Washington, DC; 2021; ISBN978-1-61537-344-4; pp.288 ; \$68 (paperback)}

\author{
Mary K. Morreale ${ }^{1}$ (D)
}

Received: 23 April 2021 / Accepted: 27 April 2021 / Published online: 10 May 2021

(C) Academic Psychiatry 2021

As medical student and resident education evolves with changes in our culture, there is an increasing amount of information to consider. How does technology impact our learners and the curricula we develop? What do regulatory agencies expect? How should we approach diversity and inclusion and learner wellness? Psychiatric educators have an increasingly greater breadth of responsibility and likely need guidance navigating the current landscape. The second edition of the Handbook of Psychiatric Education, edited by Dr. Donna M. Sudak, is designed to be an important part of that support.

Containing 15 chapters written by experts across the USA, Handbook of Psychiatric Education is divided into three parts, the first covering general topics including adult learning, professionalism, wellness, mentoring and supervision, enhancement of diversity and inclusion in training, and developing a scholarly approach to education. Part II focuses specifically on medical students and includes chapters on preclinical and clerkship curricula, evaluation techniques, and residency recruitment and advising. In addition, this section contains a chapter titled "Special Considerations in Medical Student Education" that discusses an array of topics including information overload, psychological threats to students such as illness anxiety and imposter syndrome, impediments to achievement (e.g., parenting and finances), and, finally, how students' personal experience with mental health treatment might impact their learning. Part III, Resident and Fellowship Education, includes chapters on curricular development and modification, accreditation, financing and administration of residency programs, evaluation of trainees, subspecialty training in psychiatry, and faculty development.

Mary K. Morreale

mmorreale@med.wayne.edu

1 Wayne State University, Detroit, MI, USA
As a whole, Handbook of Psychiatric Education contains a wealth of important resources. For instance, the chapter on residency curriculum includes mention of seminal publications, a description of multiple organizations involved in curriculum development, and a list of suggested texts, and the chapter on professionalism has an excellent table of assessment instruments. Several chapters have vignettes, which bring the material to life and enhance interest, and one (Special Considerations in Medical Student Education) provides questions for reflection. Each chapter is written concisely and concludes with key points and a robust list of references.

Although there are areas of the book that are somewhat superficial and identical pie charts related to diversity are printed in two sections of the text, initially in grey tone, which is difficult to decipher, and later in color, these problems do not diminish the utility of this book. As the editor notes in the introduction to this volume, it is clear that the "authors' enthusiasm for educational excellence shines through" (p. xiii). Handbook of Psychiatric Education is certainly worth reading and would likely be a valuable addition to the library of all medical student and residency educators and a vital resource for those with an administrative role.

\section{Declarations}

Disclosures The author states that there is no conflict of interest.

Publisher's Note Springer Nature remains neutral with regard to jurisdictional claims in published maps and institutional affiliations. 